\title{
QUANTITATIVE DETERMINATION OF DOMAIN DYANMICS PARAMETERS IN Co/Pd MULTILAYERED MAGNETO-OPTIC RECORDING MEDIA
}

\author{
Sug-Bong Choe and Sung-Chul Shin \\ Department of Physics, Korea Advanced Institute of Science and Technology, \\ Taejon 305-701, Korea.
}

\section{Introduction}

Recently, magnetization reversal has been extensively studied in a number of magnetic films and interestingly, the contrasting behaviors of wall-motion dominant reversal and nucleation dominant one have been reported among similar samples of many systems[1,2]. These reversal behaviors have been analyzed by the thermally activated relaxation model based on Fatuzzo's theory $[3,4]$ and also, compared with the theoretical predictions using micromagnetic simulations[5]. In this paper, we describe a new method to quantitatively analyze the magnetization reversal behaviors considering both the nucleation and wall-motion processes based on the time-dependent domain patterns.

\section{Model}

We modelize the time-dependent domain patterns of magnetization reversal based on circular domains formed by nucleation process and subsequently, expanded by wallmotion process as shown in FIG. 1. The expansion of the circular domain $d r$ in time $d t$ due to the wall-motion process is given by $d r=V d t$, while the number of nucleation $d n$ in time $d t$ is given by $d n=R(s-a) d t$ where

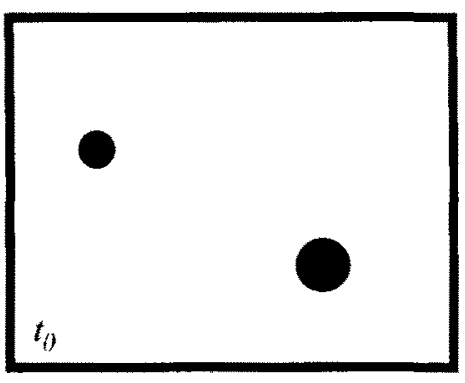

(a)

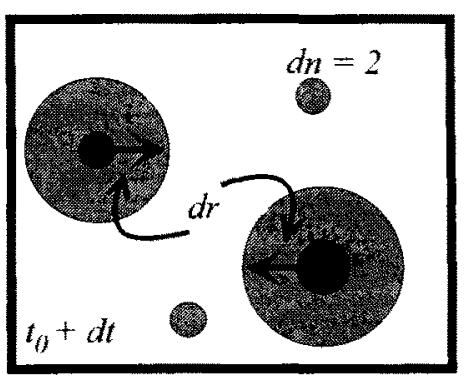

(b)

FIG. 1. A schematic illustration of the time-dependent circular domain patterns of magnetization reversal. $V$ is the wall-motion speed, $R$ is the nucleation probability per unit time and unit area, $s$ is the total area of the sample under examination, and $a$ is the reversed domain area. The expansion of the reversed domain area $d a$ is given by $d a=l d r+\pi r_{0}^{2} d n$, where the first term is caused by the wall-motion at all domain boundary of length $l$ and the second one is caused by the nucleation of circular domains having the characteristic radius $r_{0}$. On the other hand, the increment of the domain boundary length $d l$ is given by $d l=2 \pi d r+2 \pi r_{0} d n$. After substituting $d r$ and $d n$ by the previous equations and rearranging as an explicit form for $V$ and $R$, the equations are given by

$$
V=\left(a^{\prime}-r_{0} l^{\prime} / 2\right) /\left(l-\pi r_{0}\right) \text { and } R=\left(l l^{\prime} / 2 \pi-a^{\prime}\right) /\left(l-\pi r_{0}\right) r_{0}(s-a) .
$$

Thus, by measuring the reversed domain area $a(t)$ and the domain boundary length $l(t)$ from the domain patterns, one can explicitly determine the wall-motion speed $V$ and the nucleation probability $R$.

\section{Experiment}

The present method has been applied to investigate the domain reversal behavior of $\mathrm{Co} / \mathrm{Pd}$ multilayered system. A series of $\left(t_{\mathrm{Co}}-\mathrm{Co} / 11-\AA \mathrm{Pd}\right)_{10}$ samples with $t_{\mathrm{Co}}=2.0,2.5,3.0,3.5$, and 4.0 
were prepared on glass substrates by e-beam evaporation with a $2 \%$ accuracy of the sublayer thickness[6]. Magnetization reversal from the saturated state was investigated under a reversing applied field of $50 \%$ coercivity for each sample. The domain patterns were observed by a modified magneto-optic microscope capable of $\times 1,000$ magnification with an objective of 0.9 N.A.77]. A serial domain images of 7 frames per second were obtained by advanced video processing technique of the noise filtering and the black-and-white image extraction process. The reversed domain area $a(t)$ and the domain boundary length $l(t)$ were measured from the serial domain patterns by image analyzing process of the black-and-white cell-distribution counting and the edge finding. The domain boundary length $l(t)$ was calibrated due to the geometric difference between the experiment and the model. An error in the measurement caused by mapping circular domains with squared lattice, was not exceeded more than $50 \%$ even for the smallest circular domain and the error was decreased with increasing the domain radius.

\section{Result and Discussion}

$V$ and $R$ were obtained by the experimentally determined $a(t)$ and $l(t)$ under $10 \%$ reversal by setting $r_{0}=82 \mathrm{~nm}$ which corresponds to a half unit cell size of our optical observation system. In FIG. 2, we plot $V$ and $R$ with the error bars of the standard deviation from several tries as a function of the Cosublayer thickness. It is interesting to note that the mean nucleation probability is largely increased from $0.02 \mathrm{sec}^{-1} \mathrm{~m}^{-2}$ to $0.21 \mathrm{sec}^{-1} \mathrm{~m}^{-2}$ with increasing the Cosublayer thickness from $2 \AA$ to $4 \AA$, while the mean wall-motion speed is slightly decreased. Therefore, one might conclude that a sensitive change of the nucleation probability, rather than the wall-motion speed, is responsible for the contrasting reversal behaviors in the $\mathrm{Co} / \mathrm{Pd}$ multilayered system.

\section{References}

1. J. Pommier, P. Meyer, G. Ponissard, J. Ferre, P. Bruno, and D. Renard, Phys. Rev. Lett. 65, 2054(1990),

2. J. X. Shen, R. D. Kirby, Z. S. Shan, D. J. Sellmyer, and T. Suzuki, J. Appl. Phys. 73, 6418(1993).

3. E. Fatuzzo, Phys. Rev. 127, 1999(1962),

4. M. Labrune, S. Andrieu, F. Rio, and P. Bernstein, J. Magn. Magn. Mater. 80, 211(1989).

5. R. D. Kirby, J. X. Shen, R. J. Hardy, and D. J. Sellmyer, Phys. Rev. B 49, 10810(1994).
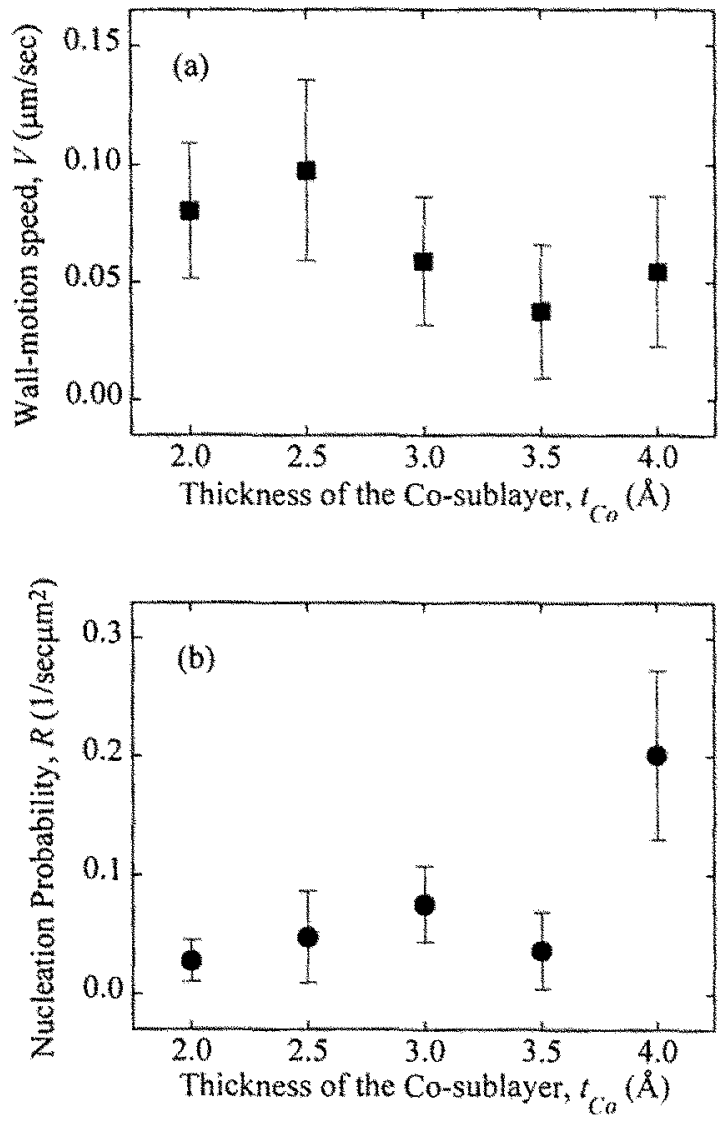

FlG. 2. The wallmotion speed $V$ and the nucleation probability $R$ of the $\left(t_{C o}-\mathrm{Co} / 11-\mathrm{A} \mathrm{Pd}\right)_{10}$ samples.

6. S.-B. Choe and S.-C, Shin, Korean J. Appl. Phys. 9, 674(1996).

7. S.-B. Choe and S.-C, Shin, J. Appl. Phys., to be published(1997). 